\title{
Evolução da Covid-19 em pacientes diabéticos em um município do sudoeste
} baiano

\author{
Evolution of Covid-19 in diabetic patients in a city in southwestern Bahia \\ Evolución de Covid-19 em pacientes diabéticos em uma ciudad del suroeste de Bahia
}

\section{Resumo}

Esse artigo objetiva identificar a evolução da Covid-19 em pacientes portadores de Diabetes Mellitus na cidade de Vitória da Conquista, Bahia, Brasil, com intuito de detectar a correlação entre os sintomas apresentados e a hospitalização desses pacientes, além de avaliar a influência de comorbidades associadas com o nível de hospitalização. Para isso realizou-se um estudo de corte transversal de natureza quantitativa. Os dados do estudo foram obtidos por meio do prontuário eletrônico inserido no Sistema de Monitoramento da Covid-19 no município de Vitória da Conquista. Desde o início da pandemia da Covid-19 em março de 2020 até o mês de agosto de 2021 foram confirmados na cidade 1770 casos de pessoas com Diabetes Mellitus contaminados pelo SARS-CoV-2. Foram analisadas características gerais como sexo, idade, raça/cor, além de sintomas e comorbidades associadas a Diabetes Mellitus, desfecho dos pacientes, e se eram profissionais de saúde. Constatou-se que apesar do sexo feminino representar uma taxa de 55\%, os pacientes do sexo masculino possuem um risco 1,8 vezes maior de evoluir para uma hospitalização. No que se refere ao índice de hospitalização, se concluiu que ele está associado á presença de outras comorbidades e no desenvolvimento de sintomas como a queda da saturação de oxigênio e dificuldades respiratórias.

Palavras-chave: Covid-19; Diabetes mellitus; Hospitalização; Pacientes; Pandemia.

\begin{abstract}
This article aims to identify the evolution of Covid-19 in patients with diabetes mellitus in the city of Vitória da Conquista, Bahia, Brazil, in order to detect the correlation between the symptoms presented and the hospitalization of these patients, in addition to evaluating the influence of comorbidities associated with the level of hospitalization. For this, a cross-sectional study of a quantitative nature was carried out. The study data were obtained through the electronic medical record inserted in the monitoring system of Covid-19 in the city of Vitória da Conquista. Since the beginning of the Covid-19 pandemic in march 2020 until the month of august 2021, 1770 cases of people with diabetes mellitus contaminated by SARS-CoV-2 were confirmed in the city. General characteristics such as gender, age, race/color, in addition to symptoms and comorbidities associated with diabetes mellitus, patient outcomes, and whether they were health professionals were analyzed. It was found that although females represent a rate of 55\%, male patients have a 1.8 times greater risk of evolving to hospitalization. With regard to the hospitalization rate, it was concluded that it is associated with the presence of other comorbidities and the development of symptoms such as a drop in oxygen saturation and breathing difficulties.
\end{abstract}

Keywords: Covid-19; Diabetes mellitus; Hospitalization; Patients; Pandemic. 


\begin{abstract}
Resumen
Este artículo tiene como objetivo identificar la evolución de Covid-19 en pacientes con Diabetes Mellitus en la ciudad de Vitória da Conquista, Bahía, Brasil, con el fin de detectar la correlación entre los síntomas presentados y la hospitalización de estos pacientes, además de evaluar la influencia de las comorbilidades asociadas al nivel de hospitalización. Para ello, se realizó un estudio transversal de carácter cuantitativo. Los datos del estudio se obtuvieron a través de la historia clínica electrónica insertada en el Sistema de Monitoreo de Covid-19 en la ciudad de Vitória da Conquista. Desde el inicio de la pandemia Covid-19 en marzo de 2020 hasta el mes de agosto de 2021, se confirmaron 1770 casos de personas con Diabetes Mellitus contaminadas por SARS-CoV-2 en la ciudad. Se analizaron características generales como sexo, edad, raza / color, además de los síntomas y comorbilidades asociadas a la Diabetes Mellitus, los resultados de los pacientes y si eran profesionales de la salud. Se encontró que aunque las mujeres representan una tasa del 55\%, los pacientes masculinos tienen un riesgo 1,8 veces mayor de progresar a la hospitalización. En cuanto a la tasa de hospitalización, se concluyó que se asocia a la presencia de otras comorbilidades y al desarrollo de síntomas como descenso de la saturación de oxígeno y dificultades respiratorias.
\end{abstract}

Palabras clave: Covid-19; Diabetes mellitus; Hospitalización; Pacientes; Pandemia.

\title{
1. Introdução
}

Enfrenta-se hoje no mundo uma das maiores crises sanitárias já vista na história, a pandemia da Covid-19 causada pelo novo coronavírus. No dia 9 de janeiro de 2020 foi identificado o patógeno que estava sendo responsável pelo surto da pneumonia viral desconhecida (Madjid et al., 2020) inicialmente chamado de novo coronavírus 19 (2019-nCoV), que posteriormente foi denominado oficialmente como coronavírus relacionado a Síndrome respiratória aguda (SARS-CoV2) pela Organização Mundial de Saúde (Zheng et al., 2020).

A nova doença ganhou o nome de Covid-19, em 11 de fevereiro de 2020, anunciado pela OMS e no dia 30 de janeiro de 2020, foi declarado também pela OMS surto da Covid-19 como uma Emergência de Saúde Pública de Interesse Internacional, que posteriormente, em 11 de março, tornou-se uma pandemia. Até o dia 19 de novembro de 2021 segundo a Organização Mundial da Saúde já haviam sido registrados 255.324.963 casos confirmados de Covid-19, incluindo 5.127.696 mortes em todo o mundo (WHO, 2021).

O SARS- CoV-2 entra nas células, pelo sistema pulmonar, por meio da ligação da proteína S (spike) do coronavírus com a enzima conversora de angiotensina (ACE2) que é um receptor de superfície celular. (Fleming et al., 2021). O período de incubação do vírus pode ser de até 21 dias, mas a média é de 7 dias após a exposição. Após esse período inicia-se o quadro clínico ou o paciente pode permanecer assintomático (Brito et al., 2020)

A transmissão se dá de pessoa para pessoa através da inoculação do vírus em membranas mucosas e também por meio do contato com materiais contaminados com secreções contendo o vírus, como gotículas de saliva. Como estratégia de prevenção, devem ser adotadas medidas de higiene e de distanciamento social a fim de diminuir a transmissão (Guinancio et al., 2020).

Os sintomas da doença Covid-19 variam, e sua apresentação vai desde assintomática até casos mais graves podendo levar a morte. Os sintomas mais comuns são: febre, tosse, fadiga, anorexia, falta de ar e mialgia. (USA, 2020). Alguns pacientes antes da febre, podem apresentar diarreia e náuseas. Devido ao dano alveolar grave, idosos e pessoas com outras doenças pré-existentes são mais propensas a desenvolver insuficiência respiratória podendo levar a internação hospitalar (Zu, et al., 2020). De modo atípico, alguns pacientes podem apresentar acidentes vasculares cerebrais, apresentações cardíacas incluindo miocardite e apresentações dermatológicas (Fleming et al., 2021).

Evidências científicas apontam que pessoas idosas e com comorbidades como hipertensão, Diabetes Mellitus, câncer, doenças pulmonares crônicas e outras, tem maior probabilidade de desenvolver uma infecção grave pela Covid-19 assim como também possuem maior probabilidade de evoluir para o óbito, sendo assim, são considerados grupos de risco (Anghebem et al., 2020). 
O Diabetes Mellitus (DM) consiste em um distúrbio metabólico caracterizado por hiperglicemia persistente, decorrente de deficiência na produção de insulina ou na sua ação, ou em ambos os mecanismos (SBD, 2019). A maior parte dos casos de DM ocorre em países em desenvolvimento, totalizando 75\% do número de casos em todo mundo, e as estimativas apontam quea maior parte do aumento dessa estatística também ocorreu nesses países (SBD, 2017). Anualmente milhares de pessoas são diagnosticadas com Diabetes Mellitus no Brasil, no ano de 2019 o país ocupava o quinto lugar entre os dez países com maior número de casos, sendo o terceiro país atrás somente dos Estados Unidos e China, com maior gasto relacionado à saúde com Diabetes totalizando 52,3 bilhões de dólares no ano de 2019 (IDF, 2019)

O paciente diabético em geral apresenta maiores riscos para infecções severas produzidas por diferentes agentes, incluindo o SARS-CoV-2. Essa associação pode ser explicada por alguns fatores, sendo eles: processo inflamatório exacerbado; alterações na coagulação e na resposta imune; eagressão direta do vírus às células das ilhotas pancreáticas que são responsáveis pela regulação glicêmica (Anghebem et al., 2020). Essas pessoas possuem uma defesa debilitada. O paciente com a DM não controlada apresenta maiores níveis de glicose no sangue, comprometendo a sua imunidade inata que é a primeira linha de defesa contra o SARS-CoV-2, o que permite a proliferação do vírus dentro do organismo (Pal \& Bhansali, 2020).

Pacientes diabéticos que fazem uso de inibidores de enzima conversora de angiotensina (IECA) ou bloqueadores do receptor angiotensina (ARB) possuem níveis altos de enzima conversora de angiotensina 2 (ACE2) no organismo nos pulmões, podendo assim, caso seja infectado pelo Covid-19, desenvolver complicações mais graves (Muniangi-Muhitu et al., 2020) visto que, o vírus SARS-CoV utiliza como receptores que medeiam sua entrada na célula, a enzima conversora de angiotensina 2 e a DPP4 (Pugliese et al., 2020).

Estudos científicos mostraram que ao comparar o paciente diabético e o não diabético internados com Covid-19, aquele que possui Diabetes Mellitus apresenta um período maior de internação hospitalar, além de gerar complicações mais graves e até mesmo uma taxa de mortalidade maior do que o indivíduo sem comorbidades (Anghebem et al., 2020).

Neste contexto, esta pesquisa tem como objetivo identificar a evolução da Covid-19 em pacientes portadores de Diabetes Mellitus na cidade de Vitória da Conquista, Bahia, Brasil, com intuito de identificar a correlação entre os sintomas apresentados, outras comorbidades e a hospitalização entre os pacientes diagnosticados com a Covid-19.

\section{Metodologia}

Trata-se de um estudo de corte transversal, com abordagem quantitativa. São denominados estudos transversais ou de corte transversal aqueles estudos que observam a situação de uma população em um momento específico, como momentâneos da realidade (Aragão, 2011).

O método quantitativo se baseia na medição objetiva e a quantificação dos resultados com o objetivo de expressar os dados a respeito de uma população através do estudo de uma pequena parte dela. Dessa forma, as pesquisas quantitativas fazem uso de uma amostra que seja representativa da população em um todo que consiga mensurar qualidades. Justifica-se como escolha desse método de pesquisa descobrir quantas pessoas de uma população específica compartilham uma característica ou um grupo de características (Zanella, 2013).

Este estudo foi realizado na rede municipal de Atenção à Saúde do município de Vitória da Conquista, BA, Brasil. A referida cidade é localizada a $509 \mathrm{~km}$ da capital Salvador. De acordo com dados do Instituto Brasileiro de Geografia e Estatística (IBGE), em 2020 a cidade tinha uma estimativa de 341.128 habitantes com densidade demográfica de 91,41 $\mathrm{hab} / \mathrm{km}^{2}$ (IBGE, 2021). 
Foi considerado como critério de inclusão para este estudo pacientes residentes na cidade de Vitória da ConquistaBA, que testaram positivo para Covid-19 no período de abril de 2020 até junho de 2021. Foram excluídos os pacientes que não possuíam, por qualquer motivo, prontuário eletrônico disponível. Os dados foram coletados do prontuário eletrônico inserido no Sistema de Monitoramento da Covid-19 no município de Vitória da Conquista, através do formulário de coleta. Com os dados obtidos na coleta, foi criado um banco de dados em planilha eletrônica do Excel®, versão 2010 Microsoft ${ }^{\circledR}$, e, posteriormente, importada para o programa estatístico SPSS Statistics (Versão 24). Com a finalidade de organizar e obter um resumo conciso dos dados foi utilizada estatística descritiva como análise de frequências (absoluta e relativa), cálculos de medidas de tendência central como média, mediana e moda, e de medidas de dispersão como variância e desvio padrão. Também foram realizadas análises inferenciais, utilizando teste Qui-Quadrado e a análise da Razão de Chances para variáveis qualitativas, adotando um nível de significância de 5\%. A pesquisa foi aprovada pelo Comitê de Ética em Pesquisa em seres humanos sob protocolo de $\mathrm{n}^{\mathrm{o}} 5.068 .864$.

\section{Resultados}

Este estudo analisou os dados de 1770 pacientes portadores de Diabetes Mellitus e diagnosticados com Covid-19 entre os meses de abril de 2020 e agosto de 2021 na cidade de Vitória da Conquista/BA. A média de idade geral foi de 59,8 anos ( \pm 14,5). Conforme o gênero a amostra foi composta por 55,2\% $(\mathrm{n}=977)$ de pacientes do sexo feminino, com idade média de 60,1 anos $( \pm 14,9$ anos $)$ e $44,8 \%(n=793)$ do sexo masculino com idade média de 59,4 anos $( \pm 14,1$ anos $)$. Foram observados ainda características étnico raciais e profissionais, conforme descrito na Tabela 1, abaixo:

Tabela 1. Descrição das características étnicos raciais e profissionais dos pacientes

\begin{tabular}{lcc}
\hline \multicolumn{1}{c}{ CARACTERÍSTICAS } & N & \% \\
\hline Raça/Cor & & \\
Não declarado & 605 & $34,2 \%$ \\
Branca & 326 & $17,9 \%$ \\
Preta & 149 & $8,4 \%$ \\
Parda & 692 & $39,1 \%$ \\
Amarela & 6 & $0,3 \%$ \\
Indígena & 2 & $0,1 \%$ \\
Profissional de Saúde & & \\
Sim & 41 & $2,3 \%$ \\
Não & 1523 & $86 \%$ \\
Não informado & 206 & $11,7 \%$ \\
\hline
\end{tabular}

Fonte: Dados da Pesquisa (2021).

Em relação aos sintomas apresentados pelos pacientes, conforme aponta Tabela 2, os de maior prevalência são tosse $(n=1160 ; 65,5 \%)$, mialgia/artralgia $(n=761 ; 43 \%)$, febre $(n=759 ; 42,9 \%)$ e cefaleia $(n=734 ; 41,5 \%)$. 
Tabela 2. Descrição dos sintomas apresentados pelos pacientes.

\begin{tabular}{|c|c|c|}
\hline CLASSIFICAÇÃO & $\mathbf{N}$ & $\%$ \\
\hline \multicolumn{3}{|l|}{ Febre } \\
\hline Sim & 759 & $42,9 \%$ \\
\hline Não & 1011 & $57,1 \%$ \\
\hline \multicolumn{3}{|l|}{ Febrícula ou Calafrio } \\
\hline Sim & 261 & $14,7 \%$ \\
\hline Não & 1509 & $85,3 \%$ \\
\hline \multicolumn{3}{|l|}{ Tosse } \\
\hline Sim & 1160 & $65,5 \%$ \\
\hline Não & 610 & $34,5 \%$ \\
\hline \multicolumn{3}{|l|}{ Dor de Garganta } \\
\hline Sim & 493 & $27,9 \%$ \\
\hline Não & 1277 & $72,1 \%$ \\
\hline \multicolumn{3}{|l|}{ Coriza } \\
\hline Sim & 550 & $31,1 \%$ \\
\hline Não & 1220 & $68,9 \%$ \\
\hline \multicolumn{3}{|l|}{ Congestão Nasal } \\
\hline Sim & 374 & $21,1 \%$ \\
\hline Não & 1396 & $78,9 \%$ \\
\hline \multicolumn{3}{|l|}{ Dificuldade de Respirar Leve } \\
\hline Sim & 347 & $19,6 \%$ \\
\hline Não & 1423 & $80,4 \%$ \\
\hline \multicolumn{3}{|l|}{ Dificuldade de Respirar Progressiva } \\
\hline Sim & 151 & $8,5 \%$ \\
\hline Não & 1619 & $91,5 \%$ \\
\hline \multicolumn{3}{|l|}{ Perda de Paladar ou Olfato } \\
\hline Sim & 396 & $22,4 \%$ \\
\hline Não & 1374 & $77,6 \%$ \\
\hline \multicolumn{3}{|l|}{ Déficit Urinário } \\
\hline Sim & 12 & $0,7 \%$ \\
\hline Não & 1758 & $99,3 \%$ \\
\hline \multicolumn{3}{|l|}{ Sincope } \\
\hline Sim & 20 & $1,1 \%$ \\
\hline Não & 1750 & $98,9 \%$ \\
\hline \multicolumn{3}{|l|}{$\mathrm{SpO}_{2}<94 \%$} \\
\hline Sim & 181 & $10,2 \%$ \\
\hline Não & 1589 & $89,8 \%$ \\
\hline \multicolumn{3}{|l|}{ Confusão Mental } \\
\hline Sim & 12 & $0,7 \%$ \\
\hline Não & 1758 & $99,3 \%$ \\
\hline Sinais de Cianose & & \\
\hline Sim & 3 & $0,2 \%$ \\
\hline Não & 1767 & $99,8 \%$ \\
\hline Sonolência & & \\
\hline Sim & 112 & $6,3 \%$ \\
\hline Não & 1658 & $97,3 \%$ \\
\hline Respiração Rápida ou Lenta & & \\
\hline Sim & 10 & $0,6 \%$ \\
\hline Não & 1760 & $99,4 \%$ \\
\hline Mialgia/Artralgia & & \\
\hline Sim & 761 & $43,0 \%$ \\
\hline Não & 1009 & $57,0 \%$ \\
\hline Diarreia & & \\
\hline Sim & 284 & $16 \%$ \\
\hline Não & 1486 & $84 \%$ \\
\hline Náuseas/Vômitos & & \\
\hline Sim & 216 & $12,2 \%$ \\
\hline Não & 1554 & $87,8 \%$ \\
\hline Cefaleia & & \\
\hline Sim & 734 & $41,5 \%$ \\
\hline Não & 1036 & $58,5 \%$ \\
\hline Irritabilidade/Confusão & & \\
\hline Sim & 18 & $1 \%$ \\
\hline Não & 1752 & $99 \%$ \\
\hline Adinamia (Fraqueza) & & \\
\hline Sim & 486 & $27,5 \%$ \\
\hline Não & 1284 & $72,5 \%$ \\
\hline Produção de escarro & & \\
\hline Sim & 80 & $4,5 \%$ \\
\hline
\end{tabular}




\begin{tabular}{lcc} 
Não & 1690 & $95,5 \%$ \\
Congestão Conjuntival & & \\
$\quad$ Sim & 84 & $4,7 \%$ \\
$\quad$ Não & 1686 & $95,3 \%$ \\
Dificuldade para deglutir & & \\
$\quad$ Sim & 51 & $2,9 \%$ \\
$\quad$ Não & 1719 & $97,1 \%$ \\
Linfonodos aumentados & & \\
$\quad$ Sim & 4 & $0,2 \%$ \\
$\quad$ Não & 1766 & $99,8 \%$ \\
Perda do apetite & 1 & \\
$\quad$ Sim & 1769 & $0,1 \%$ \\
Não & $99,9 \%$ \\
\hline
\end{tabular}

Fonte: Dados da Pesquisa (2021).

Além do Diabetes Mellitus, comorbidade de escolha para o recorte deste estudo, observa-se também outras comorbidades presentes na população estudada, conforme descrito na Tabela 3. As mais prevalentes entre os pacientes foram: doenças cardiovasculares, incluindo hipertensão arterial sistêmica - HAS $(n=480 ; 27,1 \%)$, seguido por obesidade ( $\mathrm{n}=149$; $8,4 \%)$ e asma $(\mathrm{n}=50 ; 2,8 \%)$.

Tabela 3. Descrição das comorbidades presentes nos pacientes.

\begin{tabular}{|c|c|c|}
\hline COMORBIDADE & $\mathbf{N}$ & $\%$ \\
\hline \multicolumn{3}{|l|}{ Doenca Cardiovascular (Incluindo HAS) } \\
\hline Sim & 480 & $27,1 \%$ \\
\hline Não & 1290 & $72,9 \%$ \\
\hline \multicolumn{3}{|l|}{ Doença Hepática } \\
\hline $\operatorname{Sim}$ & 27 & $1,5 \%$ \\
\hline Não & 1743 & $98,5 \%$ \\
\hline \multicolumn{3}{|c|}{ Doenca Neurológica Crônica ou Neuromuscular } \\
\hline Sim & 27 & $1,5 \%$ \\
\hline Não & 1743 & $98,5 \%$ \\
\hline \multicolumn{3}{|l|}{ Imunossuprimido } \\
\hline Sim & 11 & $0,6 \%$ \\
\hline Não & 1759 & $99,4 \%$ \\
\hline \multicolumn{3}{|l|}{ Doença Renal } \\
\hline Sim & 62 & $3,5 \%$ \\
\hline Não & 1708 & $96,5 \%$ \\
\hline \multicolumn{3}{|l|}{ Doença Pulmonar Crônica } \\
\hline Sim & 16 & $0,9 \%$ \\
\hline Não & 1754 & $99,1 \%$ \\
\hline \multicolumn{3}{|l|}{ Neoplasia } \\
\hline Sim & 12 & $0,7 \%$ \\
\hline Não & 1758 & $99,3 \%$ \\
\hline Obesidade & 149 & $8,4 \%$ \\
\hline Sim & 1621 & $91,6 \%$ \\
\hline Não & & \\
\hline \multicolumn{3}{|l|}{ Asma } \\
\hline Sim & 50 & $2,8 \%$ \\
\hline Não & 1720 & $97,2 \%$ \\
\hline
\end{tabular}

Fonte: Dados da Pesquisa (2021).

Ao correlacionar os sintomas apresentados pelos pacientes com o índice de hospitalizações, conforme apresentado na Tabela 4, é possível inferir que os sintomas exercem influência nos prognósticos de internação ou não do paciente. Foi observado que pacientes com saturação de oxigênio $\left(\mathrm{SpO}_{2}\right)$ menor do que $94 \%$ apresentaram um risco 54,3 vezes maior (I.C. $95 \%=29,182-101,076)$ de serem hospitalizados do que outros pacientes com saturações maiores. Também foram associados com maior risco para hospitalização os seguintes sintomas: dificuldade de respirar progressiva, com risco 25,0 (I.C. $95 \%=$ 14,907-41,953); dificuldade para respirar leve, com risco 1,7 maior (I.C. 95\% = 1,313-2,145); respiração rápida ou lenta, com 
risco 3,616 maior (I.C. $95 \%=1,016-12,868$ ) e febre com risco 1,364 maior (I.C. 95\% = 1,111-1,675). Já pacientes que apresentaram sintomas como febrícula/ calafrios, dor de garganta, coriza, congestão nasal, perda de paladar ou olfato, mialgia/ artralgia, cefaleia, adinamia (fraqueza) e congestão conjuntival apresentaram risco menor de serem hospitalizados do que outros pacientes com outros sintomas.

Tabela 4. Correlação entre os sintomas relatados e a hospitalização dos pacientes.

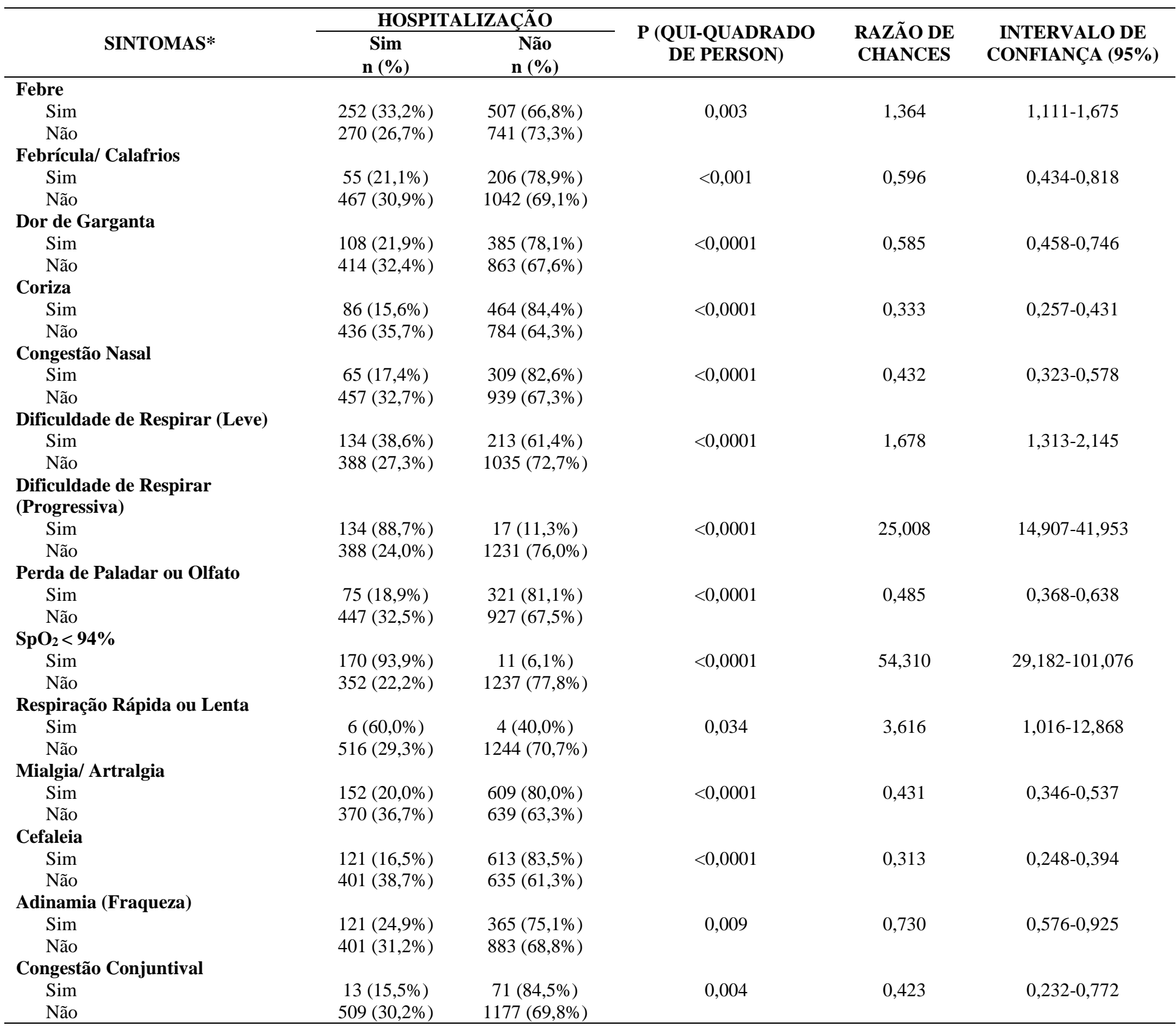

*Foram considerados somente os sintomas estatisticamente relevantes no teste Qui Quadrado, p < 0,05. Fonte: Dados da Pesquisa (2021).

Outra correlação analisada compreende as comorbidades relatadas com os índices de hospitalizações. Conforme descrito na Tabela 5, os pacientes que relataram ter doença cardiovascular (incluindo hipertensão arterial sistêmica) apresentaram risco 4,1 vezes maior (I.C. 95\% = 3,253-5,087) de ser hospitalizado do que pacientes sem essa comorbidade. O mesmo ocorreu com apresentar doença pulmonar crônica, com risco 4,043 (I.C. 95\% = 1,462-11,182); imunossuprimido com risco 4,227 (I.C. 95\% = 1,232-14,502); doença neurológica crônica/ ou neuromuscular, com risco 3,556 maior (I.C. 95\% = 
1,639-7,715) e doença renal com risco 2,317 maior (I.C. 95\% = 1,393-3,855). Foram consideradas somente as comorbidades que apresentaram diferença estatisticamente significante no teste Qui-Quadrado $(\mathrm{p}<0,05)$.

Tabela 5. Correlação entre as comorbidades relatadas e a hospitalização dos pacientes.

\begin{tabular}{|c|c|c|c|c|c|}
\hline \multirow[b]{2}{*}{ COMORBIDADES* } & \multicolumn{2}{|c|}{ HOSPITALIZAÇÃO } & \multirow{2}{*}{$\begin{array}{c}\text { P (QUI- } \\
\text { QUADRADO } \\
\text { DE PERSON) }\end{array}$} & \multirow[b]{2}{*}{$\begin{array}{l}\text { RAZÃO DE } \\
\text { CHANCES }\end{array}$} & \multirow{2}{*}{$\begin{array}{c}\text { INTERVALO DE } \\
\text { CONFIANÇA } \\
(95 \%)\end{array}$} \\
\hline & $\underset{\mathbf{n}(\%)}{\operatorname{Sim}}$ & $\begin{array}{c}\text { Não } \\
\text { n }(\%)\end{array}$ & & & \\
\hline \multirow{2}{*}{\multicolumn{6}{|c|}{$\begin{array}{l}\text { Doença Cardiovascular (Incluindo } \\
\text { HAS) }\end{array}$}} \\
\hline & & & & & \\
\hline Sim & $250(52,1 \%)$ & $230(47,9 \%)$ & $<0,0001$ & 4,068 & $3,253-5,087$ \\
\hline Não & $272(21,1 \%)$ & $1018(78,9 \%)$ & & & \\
\hline \multicolumn{6}{|l|}{$\begin{array}{l}\text { Doença Neurológica Crônica ou } \\
\text { Neuromuscular }\end{array}$} \\
\hline Sim & $16(59,3 \%)$ & $11(40,7 \%)$ & 0,001 & 3,556 & $1,639-7,715$ \\
\hline Não & $506(29,0 \%)$ & $1237(71,0 \%)$ & & & \\
\hline \multicolumn{6}{|l|}{ Imunossuprimido } \\
\hline Sim & $7(63,6 \%)$ & $4(36,4 \%)$ & 0,013 & 4,227 & $1,232-14,502$ \\
\hline Não & $515(29,3 \%)$ & $1244(70,7 \%)$ & & & \\
\hline \multicolumn{6}{|l|}{ Doença Renal } \\
\hline $\mathrm{Sim}$ & $30(48,4 \%)$ & $32(51,6 \%)$ & 0,001 & 2,317 & $1,393-3,855$ \\
\hline Não & $492(28,8 \%)$ & $1216(71,2 \%)$ & & & \\
\hline \multicolumn{6}{|l|}{ Doença Pulmonar Crônica } \\
\hline $\mathrm{Sim}$ & $10(62,5 \%)$ & $6(37,5 \%)$ & 0,004 & 4,043 & $1,462-11,182$ \\
\hline Não & $512(29,2 \%)$ & $1242(70,8 \%)$ & & & \\
\hline
\end{tabular}

*Foram consideradas somente as comorbidades estatisticamente relevantes no teste Qui Quadrado, p < 0,05. Fonte: Dados da Pesquisa (2021).

Em se tratando dos índices de hospitalização relacionados ao sexo, conforme descreve a Tabela 6, pacientes do sexo masculino tem um risco 1,8 vezes maior (I.C. $95 \%=1,441-2,177$ ) de ser hospitalizado do que pacientes do sexo feminino.

Tabela 6. Relação entre sexo e índices de hospitalização.

\begin{tabular}{|c|c|c|c|c|c|}
\hline & \multicolumn{2}{|c|}{ HOSPITALIZAÇÃO } & \multirow{2}{*}{$\begin{array}{c}\text { P (QUI- } \\
\text { QUADRADO } \\
\text { DE PERSON) } \\
\end{array}$} & \multirow[b]{2}{*}{$\begin{array}{l}\text { RAZÃO DE } \\
\text { CHANCES }\end{array}$} & \multirow{2}{*}{$\begin{array}{c}\text { INTERVALO DE } \\
\text { CONFIANÇA }(95 \%)\end{array}$} \\
\hline & $\begin{array}{c}\text { Sim } \\
\mathbf{n}(\%)\end{array}$ & $\begin{array}{c}\text { Não } \\
\text { n (\%) }\end{array}$ & & & \\
\hline \multicolumn{6}{|l|}{ SEXO } \\
\hline Masculino & $286(36,1 \%)$ & $507(63,9 \%)$ & $<0,0001$ & 1,771 & $1,441-2,177$ \\
\hline Feminino & $236(24,2 \%)$ & $741(75,8 \%)$ & & & \\
\hline TOTAL & $522(29,49 \%)$ & $1248(70,51 \%)$ & & & \\
\hline
\end{tabular}

Fonte: Dados da Pesquisa (2021).

Por fim, considerando os desfechos observados, a maioria dos pacientes diabéticos que contraíram a Covid-19 conseguiram se recuperar $(n=1547 ; 87,4 \%)$. O número de óbitos pela doença, considerando apenas pacientes com diabetes, foi de 186 (10,5\%). Considerando outras variáveis com o desfecho, como sexo, hospitalização, não foram encontradas associações estatisticamente significantes.

\section{Discussão}

A cidade de Vitória da Conquista conta com um serviço de vigilância epidemiológica que utiliza de bases científicas para desempenhar um importante papel que visa conhecer o comportamento e a história natural das doenças e seus fatores condicionantes para que o município possa intervir de maneira certa no controle e combate dessas doenças. A diretoria de Vigilância a Saúde da cidade tem a missão de organizar, controlar, e analisar o perfil epidemiológico do município e 
desenvolver estratégias com atividades de promoção e prevenção em benefício de melhorar a qualidade de vida dos habitantes e controlar os agravos à saúde dos mesmos (PMVC, 2021).

A infecção pelo SARS-CoV-2 (causador da Covid-19) em pacientes portadores de comorbidades representam um grande risco. $\mathrm{O}$ vírus infecta as células humanas por meio de uma ligação com a enzima conversora de angiotensina 2 (ECA2) presente na superfície das células. Nos pulmões a ECA2 atua degradando a angiotensina II em angiotensina 1-7, e quando a ECA1 é aumentada e a ECA2 inibida a angiotensina II tem sua degradação também inibida e vai por meio dos receptores angiotensina I e II desencadear respostas pró-inflamatórias e aumentar a secreção de aldosterona, que causa aumento da pressão arterial e aumento da permeabilidade vascular potencializando o risco de síndrome de desconforto respiratório. Pacientes com Diabetes Mellitus podem possuir aumento da ativação dos receptores de angiotensina o que associado a Covid19 pode ser mais prejudicial quando se tem um desequilíbrio prévio nessas vias, justificando o motivo destes pacientes compor o grupo de risco para essa doença (Bornstein et al., 2020). Devido a este fato, o presente trabalho teve como foco principal pacientes com essa comorbidade.

Outros fatores são estudados para explicar a relação de suscetibilidade dos portadores de Diabetes Mellitus a Covid19, como as desregulações imunes, disfunção de células fagocíticas, alteração na produção de citocinas e quimiotaxia de células de defesa, resposta imune de células $\mathrm{T}$ deficitária, dentre outras, que podem contribuir com o processo de expressa inflamação exacerbada desencadeada pelo SARS-CoV-2, o motivo de portadores de diabetes estarem suscetíveis às formas mais graves da Covid-19 não é totalmente elucidada, no entanto, é entendido que o mecanismo de ação ECA e as alterações metabólicas e imunes decorrentes da doença são significativamente importantes neste processo (Kumar et al., 2020).

A relação entre Diabetes Melittus e a Covid-19 também é discutida por Anghebem et al. (2020), o estudo aponta que paciente diabéticos possuem alta atividade de citocinas inflamatórias, assim como ocorre na Covid-19, dessa forma a associação de ambas doenças faz surgir uma característica hiperinflamatória que desencadeia desequilíbrio no processo de coagulação e fibrinólise, essas anormalidades podem favorecer um quadro pró-trombótico hipercoagulável. Segundo a OMS, $15 \%$ dos pacientes evoluem para hospitalização que necessita de oxigenoterapia e 5\% precisam ser atendidos em unidade de terapia intensiva (OMS, 2020). O presente estudo observou que existe risco aumentado de internação entre os pacientes diabéticos, visto que a taxa de hospitalização foi de 29,49\%, corroborando dessa forma com os estudos apresentados anteriormente onde avaliam a comorbidade Diabetes Mellitus como grupo de risco para Covid-19.

Este estudo observou uma prevalência de Covid-19 em mulheres diabéticas, com idade média de 60,1 de raça/cor parda. A diabetes é uma das principais comorbidades encontradas em pacientes diagnosticados com Covid-19, em uma análise de 191 pacientes infectados com o vírus destacou a diabetes como patologia recorrente entre o grupo (Zou et al., 2020).

Qian et al. (2020) mostrou que mulheres apresentam uma maior suscetibilidade ao Covid-19 assim como descrito nesse estudo, no entanto, a proporção de casos graves e críticos, bem como sua letalidade são significativamente menores nas mulheres do que em homens. Ao considerarmos pacientes diabéticos, o perfil tende a ser o mesmo, porém soma-se maior chance de casos graves entres os pacientes, em decorrência da diabetes (Michelozzi et al., 2020).

No que diz respeito a comparação da taxa de hospitalização entre homens e mulheres com Covid-19, em um estudo na cidade de Veneto, na Itália onde foi avaliado 9280 pacientes positivos para SARS-CoV-2, no geral, paciente do sexo masculino apresentaram um pior resultado clínico em relação as mulheres, sendo que os homens representaram $60 \%$ da taxa de hospitalização (Montopoli et al., 2020). A justificativa para tal fato estaria relacionada aos hormônios sexuais, onde os homens possuem o androgênio que torna o paciente mais susceptível a avançar para uma estado mais grave da doença, necessitando de hospitalização, pois a testosterona, que é um tipo de androgênio, desempenha um papel regulador positivo que leva ao aumento da expressão de TMPRSS2, que por sua vez favorece a entrada viral de SARS-CoV-2, fator este que induz a quantidade de casos graves nos pacientes deste sexo, já as mulheres, possuem como hormônio o estrogênio que poderia ter um efeito protetor 
contra a Covid-19. Tal resultado corrobora com os achados nessa pesquisa, visto que entre os pacientes diabéticos o sexo masculino apresentou um risco 1,8 vezes maior para hospitalização (Reis, et al., 2021).

Não foi observado um efeito causal entre raça/cor e pacientes diabéticos com Covid-19, são necessárias mais análise a fim de verificar a influência dessa variável. Adegunsoye et al. (2021) identificou maiores chances de pessoas negras contraírem o vírus em relação a brancos e pardos, não sendo considerado a presença ou não da Diabetes Mellitus na análise.

$\mathrm{Na}$ análise dos sintomas, este recorte observou que os mais frequentes foram tosse, mialgia/artralgia, febre e cefaleia. A sintomatologia da Covid-19 parecer ser semelhante em pacientes diabéticos ou não, esses sintomas são característicos da doença, no entanto se assemelha ao de síndromes gripais, o que os tornam inespecífico para diagnóstico, servindo somente como base para suspeita clínica, ademais, a análise do perfil sintomatológico de 191 pacientes com a doença na cidade de Wuhan - China, também demostrou os sintomas febre, tosse e dispneia como os mais recorrentes (Zou et al., 2020).

$\mathrm{O}$ presente estudo relacionou os sintomas saturação de oxigênio $\left(\mathrm{SpO}_{2}\right)$, dificuldade de respirar progressiva, respiração rápida ou lenta, dificuldade de respirar leve e febre, com as maiores chances de hospitalização, respectivamente. Os pacientes diabéticos têm maiores chances de desenvolver sintomas graves da Covid-19, que em grande maioria cursam para o desenvolvimento de sintomas respiratórios, como o surgimento da síndrome respiratória aguda grave (SRAG), esses sintomas necessitam de intervenção médica imediata, monitorização e acompanhamento contínuo, o que justificam sua maior probabilidade de hospitalização (Bastos et al., 2020). A associação destes sintomas aos portadores de diabetes positivados para Covid-19 é relacionado ao quadro de inflamação disseminado, advindos do processo imunológico da diabetes, acrescida pela alta taxa de citocinas inflamatórias pela infecção do vírus, processo que se desencadeia principalmente nos pulmões (Simão et al., 2021).

Foi observado no presente estudo que a junção de diabetes a outra comorbidade: doenças cardiovasculares (incluindo HAS), doença pulmonar crônica, doença neurológica crônica/ ou neuromuscular, e doença renal, está relacionada com as maiores chances de hospitalização, respectivamente. Uma pesquisa realizada no Estado do Espírito Santo, Brasil, onde foram investigados 104.384 casos de Covid-19 identificou que a porcentagem de internação de pessoas que apresentam somente uma comorbidade é menor quando comparada aos pacientes com multimorbidade, sendo de 12,99\%; e de 28,32\% respectivamente (Mascarello et al., 2021). A presença de multimorbidade diminui as chances de desfecho positivo pela metade, verificou-se que a associação de diabetes/hipertensão/doença renal; diabetes/hipertensão/obesidade e diabetes/hipertensão correspondem aos menores níveis de sobrevivência da Covid-19, pois a multiborbidade possibilita maior risco de intercorrências clínicas (Bustos-Vazquez \& Padilla-González, 2021).

A presença de comorbidades foi identificada como sendo o fator com maior efeito para o desfecho de óbitos por Covid-19, aumentando o risco de óbito em 9,44 vezes em comparação aos indivíduos sem comorbidade (Galvão \& Roncalli, 2020). Em um estudo realizado no estado da Bahia com pacientes positivos para Covid-19, a taxa de letalidade em indivíduos sem comorbidades foi de $2 \%$, já em pessoas com diabetes, este percentual aumentou consideravelmente, representando $12 \%$ (Carneiro, et al., 2020). Nesta pesquisa observou uma taxa de letalidade considerando apenas os pacientes diabéticos de 10,5\%, dessa forma, tal resultado está de acordo com os achados nos estudos anteriores.

Conforme mostra Bode et al. (2020) a principal causa que infere no desfecho destes pacientes é associada ao controle glicêmico prévio dos pacientes e as medidas médicas tomadas na hospitalização, pacientes diabéticos e portadores da infecção que possuíam índices glicêmicos não controlados apresentaram maiores períodos de hospitalização e maior número de óbitos, as ações rápidas da equipe de saúde que garantam melhor controle glicêmico podem contribuir significativamente com o desfecho deste grupo de pacientes.

Pacientes diabéticos positivados para Covid-19 possuem uma chance maior de óbito em relação a população em geral, risco potencial é visto quando adiciona-se o fator idade, pois ocorre aumento expressivo do risco para maiores de 65 anos (Wu 
\& McGoogan, 2020). Ademais, não é totalmente esclarecida a relação entre o sexo e a evolução de pacientes diabéticos com Covid-19 (Chen et al., 2020).

\section{Conclusão}

Após análise dos resultados, é possível perceber que o perfil de prevalência de Covid-19 em portadores de diabetes na cidade de Vitória da Conquista é composto em sua maioria por pessoas do sexo feminino, de raça/cor preta e com idade média de 60,1 anos. Entretanto, o maior índice de hospitalização está relacionado ao sexo masculino, à pacientes com presença de outras comorbidades e desenvolvimento de sintomas como febre, dificuldade de respirar (leve e progressiva) e a queda da saturação de oxigênio.

É possível inferir que os portadores de diabetes pertencem a um grupo de risco devido a sua maior probabilidade em desenvolver a forma grave da doença. Essa característica não é completamente elucidada, mas se relaciona com as características fisiológicas da diabetes e a etiologia da Covid-19. Apesar dos riscos a este grupo de paciente, uma parcela considerável evolui com a cura.

Ademais, o presente estudo se insere nas discussões em torno do momento epidêmico em decorrência da Covid-19, contribuindo na divulgação de informações e análise do perfil de uma população. Compreender as diversas variáveis da doença nos diferentes grupos é fundamental na elaboração de medidas de combate e da implementação de políticas eficazes de tratamento considerando as individualidades de cada paciente. Com isso se faz necessária à realização de posteriores pesquisas em grandes amostras na sociedade para que seja possível identificar quais são os fatores significantes que interferem na evolução de pacientes diabéticos com Covid-19 para que se possa intervir de maneira estratégica em benefício da vida desses pacientes.

\section{Referências}

Adegunsoye, A., Ventura, I. B., \& Liarski, V. M. (2020). Association of black race with outcomes in COVID-19 disease: A retrospective cohort study. Annals of the American Thoracic Society, 17(10), 1336-1339.

Anghebem, M. I., Rego, F. G. de M., \& Picheth, G. (2020). COVID-19 e Diabetes: a relação entre duas pandemias distintas. RBAC, 52(2). https://doi.org/10.21877/2448-3877.20200001

Aragão, J. (2013). Introdução aos estudos quantitativos utilizados em pesquisas científicas. Revista Práxis, 3(6). https://doi.org/10.25119/praxis-3-6-566

Bastos, L. S., Niquini, R. P., Lana, R. M., Villela, D. A. M., Cruz, O. G., Coelho, F. C., Codeço, C. T., \& Gomes, M. F. C. (2020). COVID-19 e hospitalizações por SRAG no Brasil: uma comparação até a 12a semana epidemiológica de 2020. Cadernos de Saude Publica, 36(4). https://doi.org/10.1590/0102-311x00070120

Bornstein, S. R., Dalan, R., Hopkins, D., Mingrone, G., \& Boehm, B. O. (2020). Endocrine and metabolic link to coronavirus infection. Nature Reviews. Endocrinology, 16(6), 297-298.

Bode, B., Garrett, V., Messler, J., McFarland, R., Crowe, J., Booth, R., \& Klonoff, D. C. (2020). Glycemic characteristics and clinical outcomes of COVID-19 patients hospitalized in the United States. Journal of Diabetes Science and Technology, 14(4), 813-821.

Bustos-Vazquez, E., \& Padilla-González, E. (2021). 1526Survival of hospitalized COVID-19 patients with multimorbidity in Hidalgo Mexico. International Journal of Epidemiology, 50(Supplement_1). 10.1093/ije/dyab168.104

Brito, S. B. P., Braga, I. O., Cunha, C. C., Palácio, M. A. V., \& Takenami, I. (2020). Pandemia da COVID-19: o maior desafio do século XXI. Vigilância Sanitária em Debate: Sociedade, Ciência \& Tecnologia (Health Surveillance under Debate: Society, Science \& Technology)-Visa em Debate, 8(2), 54-63.

Carneiro, D. O., da Silva, E. P., Oliveira, J. B. S., Peixoto, I. B., Pimenta, R. M. C., \& Oliveira, W. N. F. (2021). Prevalência de cura e óbito por covid-19 no estado da Bahia, Brasil, e sua correlação com condições médicas subjacentes. Visão Acadêmica, 21(4).

Centros de Controle e Prevenção de Doenças. (2020). Orientação clínica provisória para o manejo de pacientes com doença coronavírus confirmada (COVID19).

Chen, Y., Yang, D., Cheng, B., Chen, J., Peng, A., Yang, C., Liu, C., Xiong, M., Deng, A., Zhang, Y., Zheng, L., \& Huang, K. (2020). Clinical characteristics and outcomes of patients with diabetes and COVID-19 in association with glucose-lowering medication. Diabetes Care, 43(7), $1399-1407$. 
dos Reis, D. M., Gama, L. P. S. R., Teixeira, I. A., de Paula, F. G. P., Ferreira, N. N., Oliveira, R. F., \& Silva, C. L. (2021). A influência dos hormônios sexuais na resposta imunológica da COVID-19: uma revisão integrativa. Revista Eletrônica Acervo Saúde, 13(11), e9073-e9073.

Fleming, N., Sacks, L. J., Pham, C. T., Neoh, S. L., \& Ekinci, E. I. (2021). An overview of COVID-19 in people with diabetes: Pathophysiology and considerations in the inpatient setting. Diabetic Medicine: A Journal of the British Diabetic Association, 38(3), e14509.

Galvão, M. H. R., \& Roncalli, A. G. (2021). Fatores associados a maior risco de ocorrência de óbito por COVID-19: análise de importantes com base em casos confirmados. Revista Brasileira de Epidemiologia, 23 , e200106.

Guinancio, J. C., Sousa, J. G. M. de., Carvalho, B. L. de., Souza, A. B. T. de., Floriano, A. de A., \& Ribeiro, W. A. (2020). COVID - 19: Desafios do cotidiano e estratégias de enfrentamento frente ao isolamento social. Research, Society and Development, 9(8), e259985474.

Guan, W.-J., Ni, Z.-Y., Hu, Y., Liang, W.-H., Ou, C.-Q., He, J.-X., Liu, L., Shan, H., Lei, C.-L., Hui, D. S. C., Du, B., Li, L.-J., Zeng, G., Yuen, K.-Y., Chen, R.-C., Tang, C.-L., Wang, T., Chen, P.-Y., Xiang, J., China Medical Treatment Expert Group for Covid-19. (2020). Clinical characteristics of Coronavirus disease 2019 in China. The New England Journal of Medicine, 382(18), 1708-1720.

Instituto Brasileiro de Geografia e Estatisticas - IBGE. (2021). Cidades e Estados/Vitória Da Conquista. ttps://www.ibge.gov.br/cidades-eestados/ba/vitoriada-conquista.html

International Diabetes Federation. (2019). IDF Diabetes Atlas. Inís Communication. https://www.diabetes.org.br/profissionais/images/diretrizes-completa2019-2020.pdf

Kumar, A., Arora, A., Sharma, P., Anikhindi, S. A., Bansal, N., Singla, V., Khare, S., \& Srivastava, A. (2020). Is diabetes mellitus associated with mortality and severity of COVID-19? A meta-analysis. Diabetes \& Metabolic Syndrome, 14(4), 535-545.

Madjid, M., Safavi-Naeini, P., Solomon, S. D., \& Vardeny, O. (2020). Efeitos potenciais dos coronavírus no sistema cardiovascular: uma revisão. JAMA cardiology, 5 (7), 831-840.

Zu, Z. Y., Jiang, M. D., Xu, P. P., Chen, W., Ni, Q. Q., Lu, G. M., \& Zhang, L. J. (2020). Doença por coronavírus 2019 (COVID-19): uma perspectiva da China. Radiologia , 296 (2), E15-E25.

Mascarello, K. C., Vieira, A. C. B. C., Souza, A. S. S. de., Marcarini, W. D., Barauna, V. G., \& Maciel, E. L. N. (2021). Hospitalização e morte por COVID19 e sua relação com determinantes sociais da saúde e morbidades no Espírito Santo: um estudo transversal. Epidemiologia e Servicos de Saude: Revista Do Sistema Unico de Saude Do Brasil, 30(3). https://doi.org/10.1590/s1679-49742021000300004

Michelozzi, P., de'Donato, F., Scortichini, M., De Sario, M., Noccioli, F., Rossi, P., \& Davoli, M. (2020). Mortality impacts of the coronavirus disease (COVID-19) outbreak by sex and age: rapid mortality surveillance system, Italy, 1 February to 18 April 2020. Euro Surveillance : Bulletin Europeen Sur Les Maladies Transmissibles [Euro Surveillance : European Communicable Disease Bulletin], 25(19). https://doi.org/10.2807/1560-7917.ES.2020.25.19.2000620

Montopoli, M., Zumerle, S., Vettor, R., Rugge, M., Zorzi, M., Catapano, C. V., \& Alimonti, A. (2020). Terapias de privação de andrógenos para câncer de próstata e risco de infecção por SARS-CoV-2: um estudo de base populacional ( $\mathrm{N}=4532)$. Annals of Oncology, 31 (8), $1040-1045$.

Muniangi-Muhitu, H., Akalestou, E., Salem, V., Misra, S., Oliver, N. S., \& Rutter, G. A. (2020). Covid-19 and diabetes: A complex bidirectional relationship. Frontiers in Endocrinology, 11, 582936.

Pal, R., \& Bhansali, A. (2020). COVID-19, diabetes mellitus and ACE2: The conundrum. Diabetes Research and Clinical Practice, $162(108132), 108132$.

Prefeitura Municipal de Vitória da Conquista - PMVC. (2021). Saúde. https://www.pmvc.ba.gov.br/saude/

Pugliese, G., Vitale, M., Resi, V., \& Orsi, E. (2020). Is diabetes mellitus a risk factor for COronaVIrus Disease 19 (COVID-19)? Acta Diabetologica, 57(11), 1275-1285.

Qian, J., Zhao, L., Ye, R.-Z., Li, X.-J., \& Liu, Y.-L. (2020). Age-dependent gender differences in COVID-19 in mainland China: Comparative study. Clinical Infectious Diseases: An Official Publication of the Infectious Diseases Society of America, 71(9), 2488-2494

Simão, M. C. S. A., Oliveira, J. F. C. e., Rodrigues, E. C., Oliveira, F. T. de., Araujo, J. M. B. G. de., Caldeira, J. R. F., \& Lopes, A. G. (2021). Complicações pulmonares em pacientes diabéticos com infecção por covid-19 / Pulmonary complications in diabetic patientes with covid-19 infection. Brazilian Journal of Health Review, 4(2), 4636-4646.

Sociedade Brasileira de Diabetes. (2017). Diretrizes da Sociedade Brasileira de Diabetes 2017-2018. Clannad. https://edisciplinas.usp.br/pluginfile.php/4232401/mod_resource/content/2/diretrizes-sbd-2017-2018\%281\%29.pdf

Sociedade Brasileira de Diabetes. (2019). Diretrizes da Sociedade Brasileira de Diabetes 2019-2020. Clannad. https://www.diabetes.org.br/profissionais/images/DIRETRIZES-COMPLETA-2019-2020.pdf

Souza, A. S. R., Amorim, M. M. R., Melo, A. S. de O., Delgado, A. M., Florêncio, A. C. M. C. da C., Oliveira, T. V. de, Lira, L. C. S., Sales, L. M. dos S., Souza, G. A., Melo, B. C. P. de, Morais, I., \& Katz, L. (2021). Errata: General aspects of the COVID-19 pandemic. Revista Brasileira de Saúde Materno Infantil, 21(suppl 2), 565-565.

Souza, L., Randow, R., \& Sivieiro, P. (2020). Reflexões em tempos de COVID-19: diferenciais por sexo e idade. Comunicação em Ciências da Saúde, 31, 7583.

Ten-Caten, F., Gonzalez-Dias, P., Castro, Í, Ogava, R. L. T., Giddaluru, J., Silva, J. C. S., Martins, F., Gonçalves, A. N. A., Costa-Martins, A. G., Araujo, J. D., Viegas, A. C., Cunha, F. Q., Farsky, S., Bozza, F. A., Levin, A. S., Pannaraj, P. S., de Silva, T. I., Minoprio, P., Pinheiro da Silva, F., \& Nakaya, H. I. (2021). In-depth analysis of laboratory parameters reveals the interplay between sex, age, and systemic inflammation in individuals with COVID-19. International Journal of Infectious Diseases: IJID: Official Publication of the International Society for Infectious Diseases, 105, 579-587. 
Research, Society and Development, v. 10, n. 16, e221101623810, 2021

(CC BY 4.0) | ISSN 2525-3409 | DOI: http://dx.doi.org/10.33448/rsd-v10i16.23810

World Health Organization - WHO. (2021). Coronavirus (COVID-19) Dashboard.

Wu, Z., \& McGoogan, J. M. (2020). Characteristics of and important lessons from the Coronavirus disease 2019 (COVID-19) outbreak in China: Summary of a report of 72314 cases from the Chinese center for disease control and prevention: Summary of a report of 72314 cases from the Chinese center for disease control and prevention. JAMA: The Journal of the American Medical Association, 323(13), 1239-1242.

Zanella, L. $\quad$ C. H. $\quad$ (2013). Metodologia de

http://arquivos.eadadm.ufsc.br/EaDADM/UAB_2014_2/Modulo_1/Metodologia/material_didatico/Livro\%20texto\%20Metodologia\%20da\%20Pesquisa.pdf

Zheng, YY, Ma, YT, Zhang, JY e Xie, X. (2020). COVID-19 e o sistema cardiovascular. Nature Reviews Cardiology , 17 (5), $259-260$.

Zhou, F., Yu, T., Du, R., Fan, G., Liu, Y., Liu, Z., Xiang, J., Wang, Y., Song, B., Gu, X., Guan, L., Wei, Y., Li, H., Wu, X., Xu, J., Tu, S., Zhang, Y., Chen,

H., \& Cao, B. (2020). Clinical course and risk factors for mortality of adult inpatients with COVID-19 in Wuhan, China: a retrospective cohort study. Lancet, 395(10229), 1054-1062. 